\title{
Augmentation de la sensibilité des tumeurs mammaires aux anthracyclines par l'acide docosahexaénoïque en intervention nutritionnelle chez le rat
}

Oléagineux, Corps Gras, Lipides. Volume 7, Numéro 1, 107, Janvier - Février 2000, Dossier : actes des Journées Chevreul "Corps gras, nutrition et santé, questions d'actualité" (Bordeaux, Pessac)

Auteur(s) : Loïc de PONCHEVILLE, Emmanuelle GERMAIN, Jérôme MONTHARU, Philippe BOUGNOUX, Laboratoire de biologie des tumeurs, EA 2103, Université François-Rabelais, Tours.

\section{ARTICLE}

Les données actuelles suggèrent que les acides gras polyinsaturés (AGPI) augmentent la sensibilité des tumeurs mammaires à certains agents anti-cancéreux générant un stress oxydant, suggérant l'implication de la lipoperoxydation dans ce phénomène.

Nous avons montré, sur des cellules tumorales mammaires humaines in vitro, que l'activité cytotoxique de la doxorubicine, drogue anti-tumorale générant un stress oxydant, était augmentée par les AGPI n-3 ou n-6, en présence d'agent pro-oxydant (couple REDOX ascorbate/ménadione), alors qu'elle était supprimée par l'addition d'agent anti-oxydant (alpha-tocophérol) aux AGPI. L'association DHA (acide docosahexaénoïque, 22:6n-3) en présence d'agent pro-oxydant est la plus efficace à augmenter la formation de peroxydes et l'activité cytotoxique de la doxorubicine, suggérant l'implication de la peroxydation lipidique dans le phénomène observé. Puis, nous avons examiné le rôle des AGPI n-3 et de leurs interactions sur la chimiosensibilité à l'épirubicine, drogue anti-tumorale générant un stress oxydant, de tumeurs mammaires chimio-induites par le N-nitroso$\mathrm{N}$-méthyl-urée (NMU) chez le rat. Nous avons observé que, dans le groupe de rats ayant reçu un apport d'huiles de sardine riches en AGPI n-3 (DHA et EPA), en présence du couple pro-oxydant ascorbate/ménadione, la croissance des tumeurs mammaires était inhibée par l'épirubicine, alors qu'elle n'était pas affectée chez les rats ayant reçu des huiles de sardine en présence d'un agent antioxydant. Ces données indiquent qu'il est possible d'augmenter par voie nutritionnelle (huiles de poissons + agents pro-oxydants) la chimiosensibilité de tumeurs mammaires à l'épirubicine, et suggèrent l'implication de la peroxydation lipidique dans cet effet.

Il est nécessaire de déterminer le composé actif des huiles marines responsable du phénomène observé. De nombreuses études expérimentales in vitro et in vivo ont montré que le DHA était le meilleur substrat pour les réactions de peroxydation. À ce titre, nous avons examiné l'effet spécifique du DHA, dans des conditions d'oxydation contrôlée, sur la sensibilité à l'épirubicine des tumeurs mammaires induites par le NMU chez la rate. Cette étude a porté sur 48 rates Sprague-Dawley, qui ont reçu une injection unique de NMU. Les rates ont été séparées en quatre groupes nutritionnels : régime contrôle (huile de palme, $8 \%$, ajouté), régime DHA ( $8 \%$, ajouté), régime oxydant (DHA + oxydants), régime anti-oxydant (DHA + vitamine E). Dès que l'aire de la première tumeur (tumeur cible), calculée à partir du produit des plus larges diamètres, a atteint $1,5 \mathrm{~cm}^{2}$ de diamètre, une injection intra-péritonéale d'épirubicine a été effectuée $(2,5 \mathrm{mg} / \mathrm{kg})$, une fois par semaine sur une période totale de 6 semaines. Dans chaque lot, 8 rates ont constitué le sous-groupe des rates 
expérimentales et ont reçu les injections d'épirubicine, et 4 rates ont constitué le sous-groupe des rates témoins et ont reçu des injections de solution saline. Nous avons pu montrer que l'apport alimentaire de DHA dans notre modèle a permis l'augmentation de la sensibilité de ces tumeurs à l'épirubicine. Cet effet a été supprimé par l'apport d'agent anti-oxydant associé au DHA mais n'a pas été amélioré par l'apport d'agents pro-oxydants. Ces données suggèrent que le DHA est le composé actif des huiles marines, et montrent le rôle déterminant des composés anti-oxydants dans son action.

En conclusion, ces données indiquent qu'il est possible d'augmenter par voie nutritionnelle (DHA) la chimiosensiblité de tumeurs mammaires à l'épirubicine. II est nécessaire d'approfondir les études expérimentales de type intervention nutritionnelle, afin de définir précisément les conditions menant à une augmentation de la sensibilité des tumeurs mammaires aux drogues anti-tumorales générant un stress oxydant. 